\title{
Three-Dimensional Cell Culture Conditions Affect the Proteome of Cancer-Associated Fibroblasts
}

\author{
Regine C. Tölle, ${ }^{\dagger, \ddagger, \S}$ Cedric Gaggioli, ${ }^{\perp}$ and Jörn Dengjel ${ }^{*, \dagger, \ddagger \odot}$ \\ ${ }^{\dagger}$ Department of Biology, University of Fribourg, Chemin du Musée 10, 1700 Fribourg, Switzerland \\ ${ }^{\ddagger}$ Department of Dermatology, Medical Center University of Freiburg, Hauptstr. 7, 79104 Freiburg, Germany \\ ${ }^{\S}$ Faculty of Biology, University of Freiburg, Schänzlestr. 1, 79104 Freiburg, Germany \\ ${ }^{\perp}$ INSERM U1081, CNRS UMR7284, Institute for Research on Cancer and Aging, Nice (IRCAN), University of Nice Sophia, \\ Antipolis, Medical School, 28 Avenue Valombrose, 06107 Nice, France
}

\section{Supporting Information}

\begin{abstract}
In vitro cell culture systems are an invaluable tool for cell biological research to study molecular pathways and to characterize processes critical in human pathophysiology. However, the experimental conditions in two-dimensional (2D) cell cultures often differ substantially from the in vivo situation, which continuously raises concerns about the reliability and conferrability of the obtained results. Threedimensional (3D) cell cultures have been shown to closer mimic in vivo conditions and are commonly employed, for

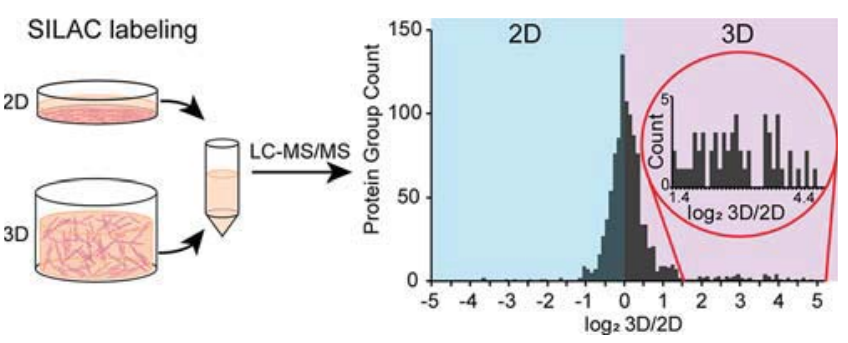
example, in pharmacological screens. Here, we introduce a 3D

cell culture system based on a mixture of collagen I and matrigel amenable to stable isotope labeling by amino acids in cell culture (SILAC) and quantitative mass spectrometry-based proteomics analyses. We study the extra- and intracellular proteomic response of skin fibroblast isolated from healthy volunteers in comparison to cancer-associated fibroblasts (CAF) on 3D culture conditions. Both, control cells and CAF, change their proteomic composition based on the culture conditions. Critically, cell type differences observed in $2 \mathrm{D}$ are often not preserved in 3D, which commonly closer resemble phenotypes observed in vivo. Especially, extracellular matrix and plasma membrane proteins are differentially regulated in $2 \mathrm{D}$ versus $3 \mathrm{D}$.
\end{abstract}

KEYWORDS: 3D, matrigel, collagen, fibroblasts, skin, cancer, mass spectrometry, bottom-up proteomics, CAF, extracellular matrix

\section{INTRODUCTION}

Biomedical science profited enormously from the introduction of in vitro tissue cultures, which allow routine analysis and perturbation of cell biological processes. Commonly, mammalian cells are kept in two-dimensional (2D) cultures using coated tissue flasks or dishes made of plastic. Although 2D cell cultures have well proven their usefulness, purists still claim that "cells grown on plastic" do not allow substantial insights into in vivo processes and pathways, advocating the use of diverse model organisms. Especially, new drug candidates still have to be tested extensively in preclinical animal models. However, animal models also present diverse pitfalls. It is now widely recognized that due to interspecies variations, animal models often do not recapitulate all aspects of human diseases, hampering successful drug development. ${ }^{1}$ One in vitro technique that can bridge the wide gap between human $2 \mathrm{D}$ cell cultures and animal models and also address distinctions of species is three-dimensional (3D) human cell culture. Especially in drug development and testing, 3D cell culture models hold great promise. ${ }^{1}$

In cancer research, several studies showed that $3 \mathrm{D}$ human cell cultures closer mimic the in vivo situation compared to $2 \mathrm{D}$ cultures. $^{2-4}$ This could be especially true for the study of the role of the tumor stroma, that is, the role of cancer-associated fibroblasts (CAF), the major stromal cell type, involved in tumor progression. ${ }^{5} \mathrm{CAF}$ were shown to remodel the protein composition of the extracellular matrix (ECM) as well as its physicochemical properties. ${ }^{6,7}$ CAF are stimulated by inflammatory conditions often observed in solid tumors and represent activated fibroblasts having similarities with woundassociated fibroblasts. ${ }^{8,9}$ Critically, CAF were shown to promote tumor growth by secretion of growth factors and hormones $^{10}$ and to promote cancer cell invasion by ECM deformation, generating tracks that can guide migration of cancer cells. A global analysis on how CAF alter the ECM is still missing, and it is not well understood how and which ECM proteins stimulate cancer cell growth and migration. To address these fundamental questions, we aimed to develop a quantitative mass spectrometry (MS)-based proteomics protocol that allows the identification and accurate quantification of CAF proteins and of their microenvironment.

To study the cellular response to $3 \mathrm{D}$ culture conditions by proteomics approaches, mainly 3D spheroid cultures or 3D cell 
layers were used. ${ }^{11-13}$ So far, stable isotope labeling by amino acids in cell culture (SILAC)-based quantitative MS analyses ${ }^{14}$ of 3D cell cultures were performed of endothelial cells grown in matrigel using a SILAC labeled 2D spike-in ${ }^{15}$ or of SILAClabeled embryonic stem cells. ${ }^{16}$ Recently, a SILAC protocol for organoids was published obtaining $85 \%$ label incorporation after 30 days in culture. ${ }^{17}$ Critically, to our knowledge, all published studies purified cells/organoids prior MS analyses and focused on the characterization of intracellular proteomes. The protein compositions of the microenvironments were not studied. However, because of the specific role CAF play in cancer cell invasion, our aim was to establish an approach addressing both the intracellular and extracellular compartments. Thus, we developed a SILAC-based protocol, allowing the complete labeling of fibroblasts grown in a $3 \mathrm{D}$ matrix consisting of a mixture of collagen I and matrigel. Such cultures are commonly used in cell biological assays to study the capacity of fibroblasts to deform ECM, ${ }^{7,18}$ a proxy to study the promotion of cancer cell invasion by stromal cells. In the current study, we focused on the technical aspects of 3D cell cultures without addressing the heterogeneity of primary human cell types. We performed comparative expression proteomics experiments of CAF and normal human skin fibroblasts (NHF) isolated from healthy volunteers and analyzed cell type-specific responses to 3D culture conditions.

\section{EXPERIMENTAL SECTION}

Cells

NHF were isolated from the foreskin of a circumcised 4 yearold healthy boy as described previously. ${ }^{19}$ CAF were isolated from a head and neck squamous cell carcinoma as published. The study was approved by the Ethics Committee of Freiburg University and conducted according to the Declaration of Helsinki.

Stable Isotope Labeling by Amino Acids and 3D Cell Culture

For the establishment of 3D cultures, NHF and CAF were cultured and passaged in SILAC-Dulbecco's modified Eagle's medium (Thermo Fisher Scientific), supplemented with $10 \%$ dialyzed fetal bovine serum (Gibco), 1\% penicillin/streptomycin (Invitrogen), 1\% L-glutamine (PAN Biotech), $84 \mathrm{mg} \mathrm{L}^{-1} \mathrm{~L}-$ arginine (Sigma-Aldrich), $146 \mathrm{mg} \mathrm{L}^{-1}$ L-lysine (SigmaAldrich), and $125 \mathrm{mg} \mathrm{L}^{-1}$ proline (Sigma-Aldrich) for 10 days, followed by 1 week in serum-free medium. Insulin (10 $\left.\mathrm{mg} \mathrm{L}^{-1}\right)$-transferrin $\left(5.5 \mathrm{mg} \mathrm{L}^{-1}\right)$-selenium $\left(0.0067 \mathrm{mg} \mathrm{L}^{-1}\right)$ supplement (Gibco) was used as a serum substitution. In addition, fibroblasts were treated with $50 \mu \mathrm{g} \mathrm{mL}^{-1}$ ascorbate for proper collagen production. ${ }^{20}$ Finally, $5 \times 10^{5}$ fibroblasts were embedded in $1 \mathrm{~mL}$ of collagen I $\left(4.6 \mathrm{mg} \mathrm{mL}^{-1}\right)$-matrigel $\left(2.2 \mathrm{mg} \mathrm{mL}^{-1}\right)^{7}$ containing $5 \times$ SILAC DMEM and cultured for up to 5 days under serum-free conditions in the presence of insulin-transferrin-selenium and ascorbate. The $5 \times$ SILAC DMEM was made with powdered DMEM (Thermo Fisher Scientific) and $420 \mathrm{mg} \mathrm{L}^{-1}$ L-arginine, $730 \mathrm{mg} \mathrm{L}^{-1} \mathrm{~L}$-lysine, and $625 \mathrm{mg} \mathrm{L}^{-1}$ proline. For the preparation of the collagen Imatrigel matrix, $5 \times$ DMEM was diluted 1:10. Thus, the final concentration in the gel was $42 \mathrm{mg} \mathrm{L}^{-1} \mathrm{~L}$-arginine, $73 \mathrm{mg} \mathrm{L}^{-1}$ heavy L-lysine, and $62.5 \mathrm{mg} \mathrm{L}^{-1}$ proline. Every other day, medium was replaced by fresh regular SILAC medium (see above).

For $2 \mathrm{D}$ culture, $10 \mathrm{~cm}$ culture plates were coated with $5 \mathrm{~mL}$ of $20 \mu \mathrm{g} \mathrm{mL}^{-1}$ collagen I (Corning) and $27 \mu \mathrm{g} \mathrm{mL}^{-1}$ matrigel (growth factor-reduced, Corning) in $0.02 \mathrm{M}$ acetic acid for $1 \mathrm{~h}$ at $37{ }^{\circ} \mathrm{C}$ and $5 \% \mathrm{CO}_{2}$. Primary fibroblasts for the super-SILAC mix were grown in $2 \mathrm{D}$ and labeled with $42 \mathrm{mg} \mathrm{L}^{-1} \mathrm{~L}$-arginine, $73 \mathrm{mg} \mathrm{L}^{-1}$ L-lysine (Sigma-Aldrich), and $80 \mathrm{mg} \mathrm{L}^{-1}$ proline. "Heavy" L-arginine $-{ }^{13} \mathrm{C}_{6}{ }^{15} \mathrm{~N}_{4}$ (Arg10) and L-lysine- ${ }^{13} \mathrm{C}_{6}{ }^{-15} \mathrm{~N}_{2}$ (Lys8) or "medium-heavy" L-arginine- ${ }^{13} \mathrm{C}_{6}$ (Arg6) and $\mathrm{L}$ lysine $-{ }^{2} \mathrm{H}_{4}$ (Lys4) amino acids were used. The "light" label was not used. Biological replicates were obtained by swapping labels.

\section{Protein Solubilization and MS Sample Preparation}

Cells were lysed after 5 days of $2 \mathrm{D}$ or $3 \mathrm{D}$ culture in $1 \mathrm{~mL}$ of $2 \times$ RIPA buffer. ${ }^{21}$ Pellet and supernatant were separately processed for in-gel digestion. Samples were concentrated on a $10 \mathrm{kDa}$ cutoff filter (Sartorius) and supplemented with SDSPAGE loading buffer. Disulfide bonds were reduced with 1 mM DTT (Sigma-Aldrich) for $5 \mathrm{~min}$ at $95{ }^{\circ} \mathrm{C}$ and alkylated using $5.5 \mathrm{mM}$ iodoacetamide (Sigma-Aldrich) for $30 \mathrm{~min}$ at 25 ${ }^{\circ} \mathrm{C}$. The mixing ratio was determined by setup experiments: aliquots were mixed, proteins digested, and peptides quantified by MS. Samples were mixed in a 1:1 peptide ratio. Protein mixtures were separated by SDS-PAGE using 4-12\% Bis-Tris gradient gels (Invitrogen). Gel lanes were cut into 10 slices, proteins therein were digested with trypsin (Promega), and peptides were processed on STAGE tips as described. ${ }^{19}$

\section{Mass Spectrometry}

MS analyses were conducted on an LTQ Orbitrap XL and 3D cultures were also analyzed on a QExactive Plus mass spectrometer (both Thermo Fisher Scientific). For the LTQ Orbitrap XL, samples were separated by an Agilent 1200 HPLC, for the QExactive an EasyLC 1000 (Thermo Fisher Scientific) was coupled to the mass spectrometer. Chromatography was conducted with in-house packed Reprosil-Pur 120 ODS-3 (Dr. Maisch) fused silica HPLC-columns with $75 \mu \mathrm{m}$ inner diameter. Samples were applied to the column with a gradient of A ( $0.5 \%$ acetic acid) and B (0.5\% acetic acid in $80 \%$ $\mathrm{ACN}$ ) with increasing ACN proportion for peptide separation. Samples were loaded with $2 \% \mathrm{~B}$; a separation gradient was run for $100 \mathrm{~min}$ increasing the percentage of buffer B to $35 \%$. The spray voltage was $2.3 \mathrm{kV}$ with no sheath and auxiliary gas flow and an ion-transfer tube temperature of $200{ }^{\circ} \mathrm{C}\left(250{ }^{\circ} \mathrm{C}\right.$ for the QExactive). The data were acquired in the data-dependent mode and switched automatically between MS and MS/MS with a maximum of $1 \times 10^{6}$ ions in MS. The top five (top 10 for the QExactive) most abundant peptides were isolated in the linear ion trap and fragmented with $35 \%$ collision energy (25\% for the QExactive) and a target value of 5000. Parent ions with a charge state of $z=1$ and unassigned charge states were excluded from fragmentation. The mass range for the LTQ Orbitrap was set to $350-2000 \mathrm{~m} / z$ with a resolution of 60000 , for the QExactive Plus the mass range was $370-1750$ $\mathrm{m} / z$ with a resolution of 70000 .

\section{Identification and Quantification of Proteins Using MaxQuant}

Raw mass spectra data files were searched with the MaxQuant software version 1.4.1.2 $2^{22}$ using a UniProt Homo sapiens database from March 2016, containing 21033 entries. The defined fixed modification was carbamidomethylation of cysteines, whereas methionine oxidations and protein aminoterminal acetylations were set as variable modifications. Three missed cleavages were allowed for enzymatic cleavage with trypsin/P. Quantitation was done with a setting of double or 

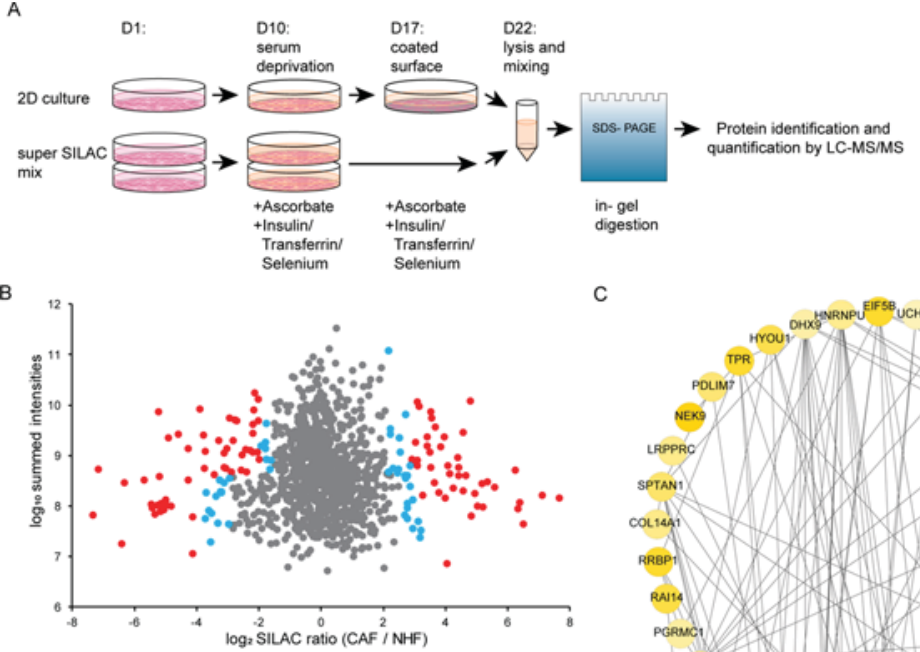

C

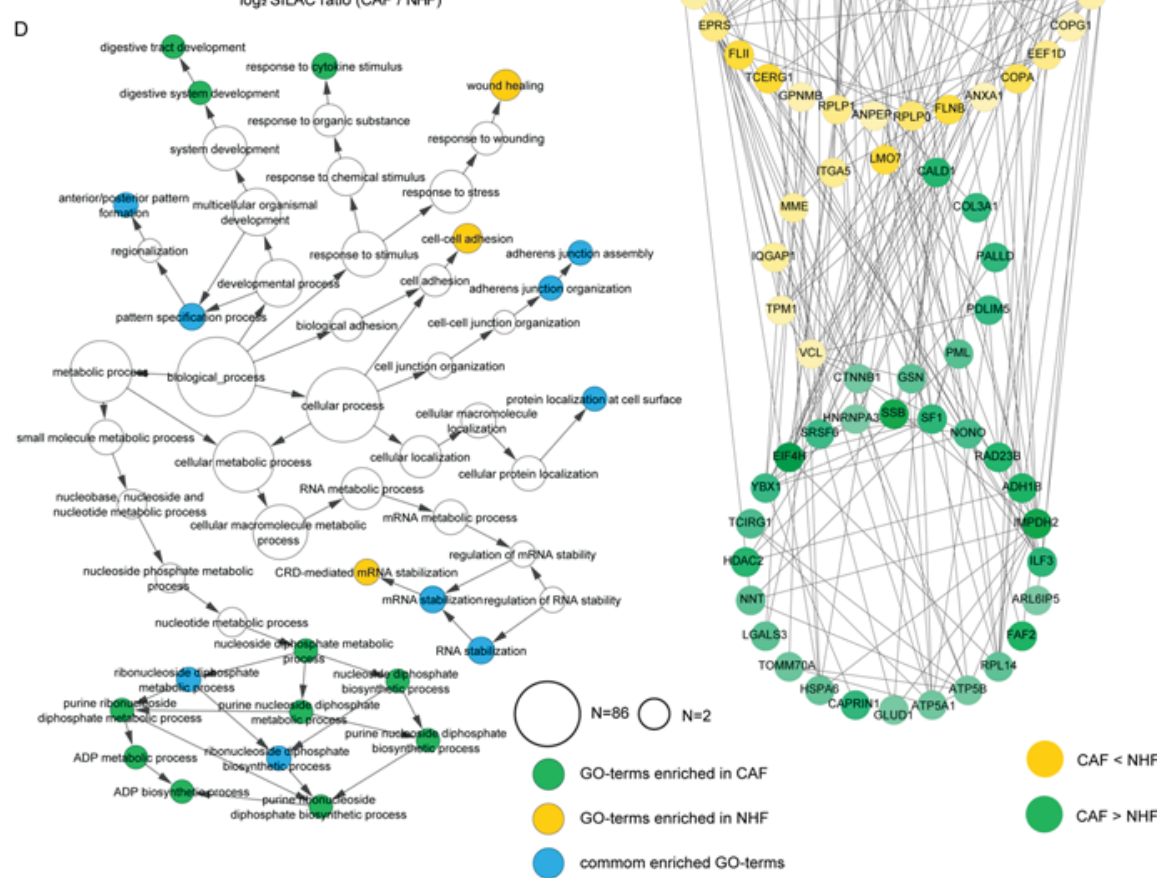

Figure 1. Quantitative proteome comparison of CAF and NHF grown in 2D. (A) SILAC labeling strategy of cells from 2D cultures. Cells were SILAC labeled for 10 days in DMEM supplemented with $10 \%$ dialyzed (d)FBS. At day 10, cells were deprived of dFBS and DMEM supplemented with ascorbate and insulin-transferrin-selenium was used for 7 days. Subsequently, cells were transferred to collagen I-matrigel coated dishes for 5 days. After lysis, cells were mixed with a super-SILAC mix and submitted to in-gel digestion. (B) Protein abundance plot comparing CAF and NHF proteomes by a SILAC approach $(n=2)$. Significantly altered proteins were determined by performing outlier tests using Significance B values generated by MaxQuant ${ }^{22}$ and Perseus. ${ }^{24}$ Regulated proteins are highlighted in blue $(p \leq 0.05)$ and in red $(p \leq 0.01)$, respectively. (C) STRING network analysis of significantly regulated proteins in CAF and NHF $(p \leq 0.01)$. (D) GO-term enrichment analysis $(p \leq 0.03$, colored circles) of significantly regulated proteins.

triple SILAC. The MS/MS tolerance was set to $0.5 \mathrm{Da}$, whereas the average mass precision of identified peptides was less than $20 \mathrm{ppm}$. FDRs of 0.01 were applied for peptide and protein identification, the minimum peptide length was set to seven, and at least one peptide was unique. For quantification, a minimum ratio count of two was chosen. Identified proteins were requantified.

MS proteomics data were deposited to the ProteomeXchange Consortium via the PRIDE $^{23}$ partner repository with the data set identifier PXD009424.

qRT-PCR

RNA was isolated with an RNA isolation kit (Quiagen) or by Tri-Reagent (Sigma) homogenization, chloroform extraction, and isopropyl alcohol precipitation. Extracted RNA was further purified with an RNeasy cleanup kit (Quiagen). One microgram of RNA was used for reverse transcription with a Quantitect RT kit (Qiagen) and the gDNA wipeout reaction to eliminate genomic DNA was performed for $10 \mathrm{~min}$ at $42{ }^{\circ} \mathrm{C}$. Primers were mixed equally at $50 \mu \mathrm{M}$. qRT-PCR was performed with a Rotor-Gene $Q$ (Quiagen). The KAPA SYBR Fast qPCR mix (KAPA Biosystems) was used for the PCR according to the manufacturer's recommendations. Double strands were separated by heating to $95{ }^{\circ} \mathrm{C}$ for 10 min; then 40 cycles were performed of $10 \mathrm{~s}$ at $95^{\circ} \mathrm{C}, 15 \mathrm{~s} 60$ ${ }^{\circ} \mathrm{C}$, and 20 s $72{ }^{\circ} \mathrm{C}$. Primer sequences were as follows: for TGM2, forward 5'-CCA ACT ACA ACT CGG CCC AT-3', reverse 5'-CTG GTC ATC CAC GAC TCC AC-3'; for TNC, 
forward 5'-ACA GGC GAG AAA GTG CCA GA-3', reverse 5'-TGT GTA TTC CGT GGC AGG CT-3'; for MYOF, forward 5'-TCA GAA CGA GAG CCG CTA CC-3', reverse 5'-TTA TCG CCG TTC GCA TCC GT-3'. Data were normalized to HPRT, which was used as internal control with the following primers: forward 5'-TGA CAC TGG CAA AAC AAT GCA-3', reverse 5'-GGT CCT TTT CAC CAG CAA GCT-3'.

\section{Western Blot}

Three-dimensional gels were dissolved in $2 \times$ RIPA buffer supplemented with phosphatase and protease inhibitors (Roche). Lysates were dissolved with a syringe and DNA was digested with $0.1 \% \mathrm{v} / \mathrm{v}$ benzonase (Emprove Bio). Samples were centrifuged for $12 \mathrm{~min}$ at $13500 \mathrm{rpm}$ at $4{ }^{\circ} \mathrm{C}$. The volume of the supernatant was reduced on a $10 \mathrm{kDa}$ cutoff filter (Sartorius) and supplemented with SDS-PAGE loading buffer. Proteins were separated by SDS-PAGE and blotted onto nitrocellulose membranes. The membranes were blocked for $30 \mathrm{~min}$ in $5 \%$ BSA in $1 \times$ TBS with $0.1 \%$ Tween- 20 . Primary antibodies were anti-GAPDH, HRP (sc-25778HRP), and anti-MYOF (sc-376879), both Santa Cruz Biotechnology. Antibodies were diluted 1:1000 in TBS-0.1\% Tween with 5\% BSA and incubated with the membranes overnight at $4{ }^{\circ} \mathrm{C}$. The HRP-conjugated sheep anti-mouse secondary antibody (GE Healthcare) was diluted 1:10 000 in 5\% BSA in TBS-0.1\% Tween and incubated with the membrane for $1 \mathrm{~h}$ at room temperature. Chemiluminescence was detected by SuperSignal West Femto Maximum Sensitivity Substrate (Thermo Scientific).

\section{Data Analyses}

Data analysis was performed using Perseus. ${ }^{24}$ Significantly regulated proteins were identified by performing outlier tests relative to the respective $\log _{2}$-transformed total data set (Significance A, $p<0.05$ ). To determine intensity-dependent outliers, Significance B $(p<0.05)$ was used. ${ }^{22}$ Generation of protein networks was done in STRING DB. ${ }^{25}$ Network analysis and GO-term statistics were done with Cytoscape 3.5.1 ${ }^{26}$ and BINGO 3.0.3. ${ }^{27}$ Calculation of ontology enrichment was conducted on the background of the entire data set. Ontology nodes with a $p$-value $\leq 0.03$ were determined as significant. For the $2 \mathrm{D}$ data set, proteins with a $p$-value $\leq 0.01$ were evaluated on the background of 1117 proteins. For 3D, proteins with a $p$-value $\leq 0.05$ were analyzed on the background of 961 proteins.

\section{RESULTS AND DISCUSSION}

\section{NHF and CAF Grown in 2D Differ in Their Proteomes}

As CAF were characterized to promote tumor cell invasion compared to $\mathrm{NHF},{ }^{7,28}$ we asked if these phenotypic differences were reflected by proteome alterations under standard 2D cell culture conditions. Using a super-SILAC approach the proteomes of NHF and CAF cultured in 2D on coated dishes were analyzed quantitatively by geLC-MS/MS (Figure 1A; see Experimental Section for details). ${ }^{29}$ Serum leads to fibroblast activation triggering wound repair responses ${ }^{30}$ and promoting expression of genes commonly expressed in tumors. ${ }^{9}$ Thus, to be able to discriminate fibroblasts activated in vivo, that is, CAF, from nonactivated fibroblasts, we implemented a serum-free cell culture strategy.

We identified 2577 proteins of which 1147 could be quantified in at least one replicate each using an LTQ-Orbitrap
XL $(n=2$, Supplementary Figure S1, Supplementary Table $\mathrm{S} 1)$. Out of the 1147 common proteins, 142 proteins were significantly differentially regulated between NHF and CAF (Figure 1B; $p \leq 0.05$ ). 74 proteins showed a significantly higher protein abundance in NHF than in CAF, whereas 68 proteins were enriched in CAF. Interestingly, 74 of the differentially regulated proteins are known to interact according to STRING DB (Figure 1C). ${ }^{31}$ We performed GO-term enrichment analyses to identify cellular pathways/ processes, which might be affected by the altered proteome composition in CAF. Proteins involved in adherens junction ( $p$ $=0.007)$, cell-cell adhesion $(p=0.01)$, wound healing $(p=$ $0.003)$, nucleotide metabolic processes $(p=0.007)$, and proteins localizing to the cell surface $(p=0.02)$ were significantly differentially regulated (Figure 1D and Supplementary Figure S2), corroborating the finding that CAF are a terminally modified cell type that can be propagated in vitro. Among others, we identified an upregulation of catenin beta-1 (CTNNB1), which was shown to be critical for CAF to guide collective cell migration. ${ }^{32}$ CTNNB1 is a key component of the canonical Wnt signaling pathway, which was linked to the proinvasive phenotype of CAF. ${ }^{33}$

Although we detected significant differences between CAF and NHF that could be linked to the tumor-promoting phenotype of CAF observed in vivo, we were wondering if we missed important information due to the limitations of our cell culture model. Therefore, we decided to establish a cell culture system that highlights in vivo relevant, phenotypic differences between CAF and NHF and, importantly, that allows the quantitative comparison of CAF and NHF proteomes.

Contraction of 3D Cell Cultures Is a Readout for Tumor Invasion-Promoting Effects of Stromal Cells

Fibroblasts can be propagated in 3D cultures based on a mixture of collagen I and matrigel. Critically, activated fibroblasts remodel and contract the gel $^{34}$ and the extent of contraction was shown to correlate with the ability of fibroblasts to promote tumor cell invasion. ${ }^{35,36}$ To not conceal phenotypic differences between CAF and NHF, fibroblast subtypes were again kept serum-free. As expected, intrinsically activated CAF contracted the matrix significantly stronger and faster than NHF (Figure 2). After 1 day, CAF showed an
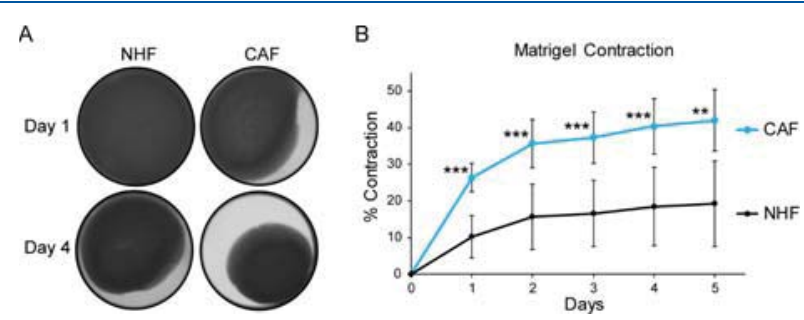

Figure 2. CAF contract 3D matrices stronger than NHF. (A) Pictures of NHF and CAF grown in $3 \mathrm{D}$ culture show the activated phenotype of CAF by (B) significantly enhanced gel contraction (NHF $n=6$, CAF $n=8$, two-tailed, homoscedastic $t$-test). $* * * p \leq 0.001, * * p \leq$ $0.01, * p \leq 0.05$.

average matrix contraction by $26 \%$, whereas NHF contracted the gel only by $10 \%(p<0.001)$. After 5 days, CAFs reduced the gels by $42 \%$ of its original size and NHF by $26 \%$ ( $p<$ $0.01)$. To identify the differentially regulated molecular mechanisms in CAF grown in such a collagen I and matrigel-based 3D culture, we adapted the culture conditions 
and established a SILAC-based quantitative proteomics approach addressing both the cellular proteome of fibroblasts and the ECM embedding the cells grown in 3D.

\section{D-SILAC for Quantitative MS Analyses}

As collagen $\mathrm{I}$ is isolated from rat-tails and matrigel from Engelbreth-Holm-Swarm mouse sarcoma cells, both products contain unlabeled proteins that may get reused by cells. In addition, both products may contain free, unlabeled amino acids, which would negatively interfere with SILAC labeling. Hence, we established a SILAC protocol based on powdered DMEM to be able to modify medium compositions at will (see Experimental Section for details). However, before analyzing label incorporation using our new setup, we comprehensively mapped the matrigel and collagen I solution proteomes to identify all proteins that potentially share peptides between mouse/rat and human orthologues and to be able to exclude these proteins for the determination of labeling efficiency. We detected 435 murine proteins in matrigel of which laminin subunit beta-1 and gamma-1, nidogen-1, vimentin, and fibrinogen gamma chain showed the highest intensities in agreement to published data. ${ }^{16} 176$ of these share common peptides with human orthologues. The purified collagen I solution contained 56 different rat proteins with the highest intensities for collagen alpha-1 and alpha- 2 chains, vimentin and actin, and all of these share peptides with human orthologues (Supplementary Table S2). For the determination of metabolic labeling efficiencies, we excluded respective proteins.

Using an analogous cell culture protocol as for $2 \mathrm{D}$, we performed 3D-SILAC-based quantitative proteomics analyses. After 10 days in full SILAC medium and an additional 7 days in serum-deprived medium in $2 \mathrm{D}$ culture, fibroblasts were transferred for 5 days to serum-deprived 3D cultures (Figure $3 \mathrm{~A}$ ). Incubation with $42 \mathrm{mg} \mathrm{L}^{-1}$ heavy L-arginine and $73 \mathrm{mg}$ $\mathrm{L}^{-1}$ heavy L-lysine showed a median labeling efficiency of 90.3\% and 93.7\%, respectively (Supplementary Figure S3). To optimize label incorporation, we cultured fibroblasts in DMEM
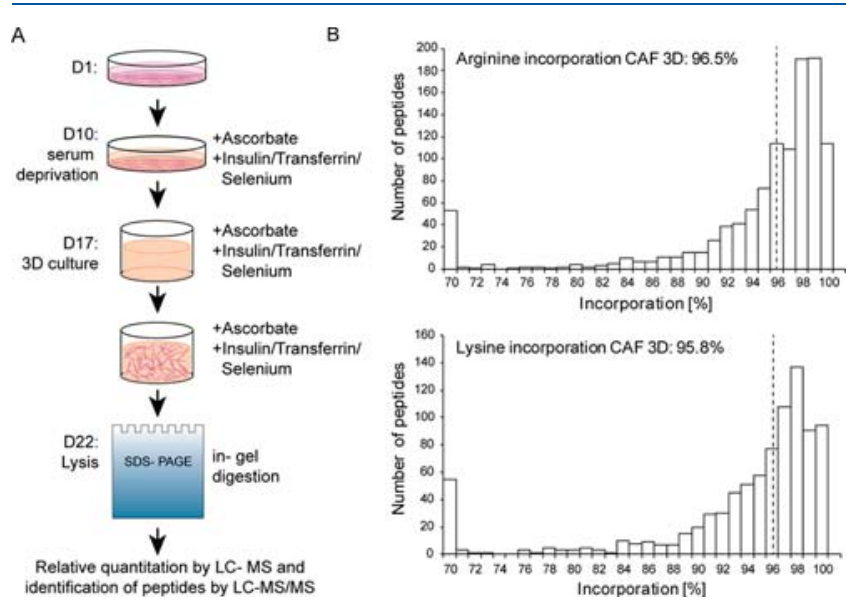

Figure 3. 3D-SILAC setup. (A) SILAC labeling strategy for primary human fibroblasts grown in 3D culture. Fibroblasts were SILAC labeled for 10 days in full medium and further incubated for 7 days in serum-deprived medium in 2D. After 5 days in $3 \mathrm{D}$, cells were lysed and processed by geLC-MS/MS. (B) Isotopic label incorporation of CAF. The 1275 lysine- and 1130 arginine-containing peptides were analyzed for their incorporation efficiency (Arg10, Lys8) in relation to unlabeled peptides (Arg0, Lys0). The dotted lines mark the median labeling efficiencies. containing $84 \mathrm{mg} \mathrm{L}^{-1}$ heavy L-arginine, $146 \mathrm{mg} \mathrm{L}^{-1}$ heavy Llysine, and $125 \mathrm{mg} \mathrm{L}^{-1}$ proline to counteract arginine-toproline conversion. We reached $95.8 \%$ labeling efficacy of lysine- and $96.5 \%$ of arginine-containing peptides (Figure 3B), which is similar to results obtained in $2 \mathrm{D} .{ }^{37}$ Arginine-to-proline conversion was below 4\% (Supplementary Figure S3). Thus, this 3D-SILAC approach permits an accurate quantitative analysis of different cell types and their produced ECM grown in $3 \mathrm{D}$ cultures as well as a characterization of the influence of culture conditions on cellular proteomes.

Cells Grown in 3D Upregulate Proteins Involved in ECM Organization and Establishment of Cell-ECM Contacts

First, we asked which pathways were affected by $3 \mathrm{D}$ culture conditions independently of the used cell type and quantitatively compared the proteomes of CAF and NHF grown in $3 \mathrm{D}$ to respective cells grown in $2 \mathrm{D}$ cultures (Figure $4 A)$. SILAC-labeled cells grown for 5 days in $3 \mathrm{D}$ or in $2 \mathrm{D}$,

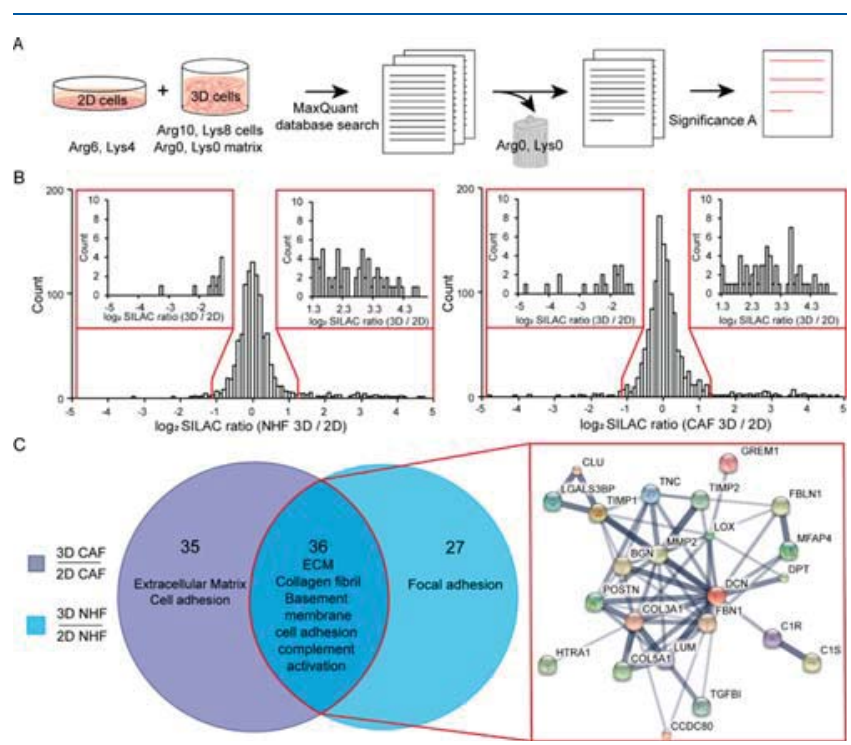

Figure 4. Comparison of $2 \mathrm{D}$ and $3 \mathrm{D}$ culture conditions. (A) Experimental workflow and data processing. MaxQuant database searches were performed using "triple SILAC" as quantification mode. Light SILAC-labeled proteins were excluded from further analysis. (B) Histogram of 3D versus 2D protein ratios of NHF (left) and CAF (right). Enlarged are $\log _{2}$ SILAC ratios smaller than -1.3 and greater than 1.3. 3D culture conditions led to an upregulation of protein subsets. (C) GO-term enrichment analysis (FDR $<0.05, \mathrm{BH}$ corrected) for significantly up- and downregulated proteins in CAF, $\mathrm{NHF}$, or in both is shown (significance A, $p<0.05, \mathrm{BH}$-corrected). A STRING DB analysis of the 36 commonly regulated proteins identified proteins known to potentially interact.

respectively, were mixed in a 1:1 peptide ratio and analyzed by LC-MS/MS. To be able to discriminate cellular peptides from 3D matrix peptides, cells were exclusively labeled by "mediumheavy" and "heavy" amino acids, whereas the "light" label was used to identify nonlabeled matrigel and collagen I peptides, which were excluded from further analysis (Figure 4A). We identified 1515 proteins of which we could quantify 1107 proteins in two replicates each (Supplementary Figure S4 and Supplementary Table S3). Interestingly, comparison of the two culture conditions showed a significant upregulation of protein populations in 3D cultures (Figure 4B; Significance A, $p<$ $0.05)$. According to a $\mathrm{GO}$ analysis, $3 \mathrm{D}$ cell cultures stimulate the expression of proteins involved in ECM organization (FDR 

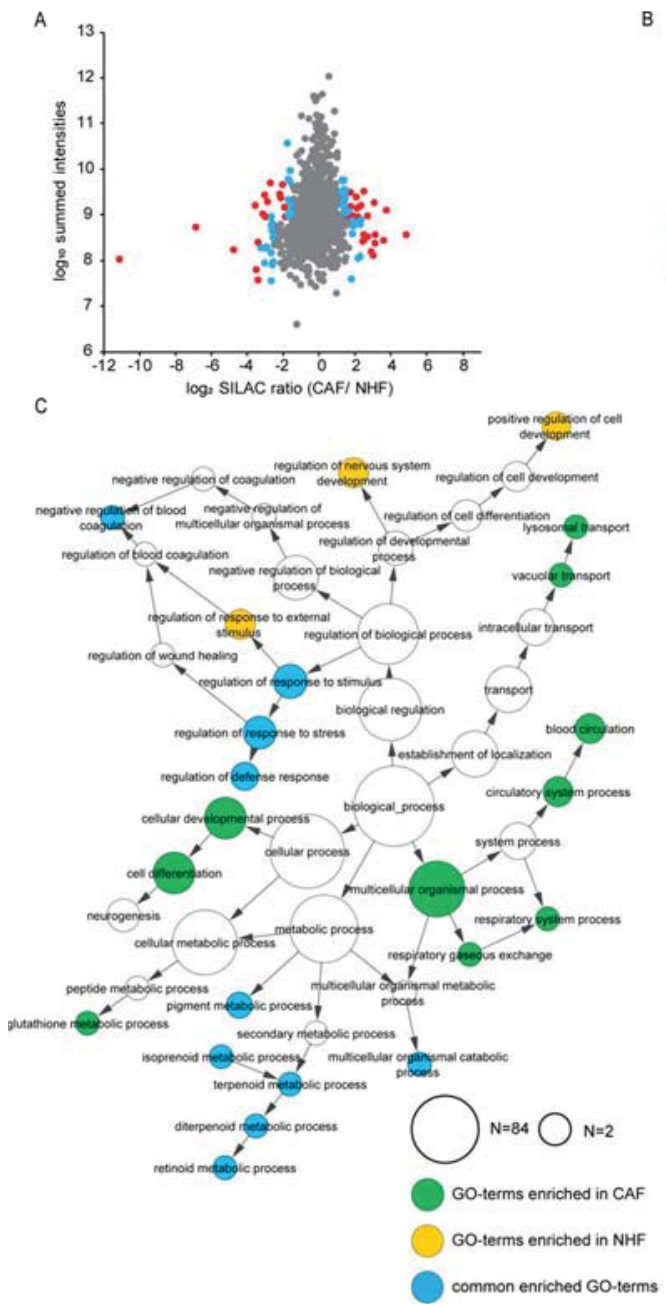

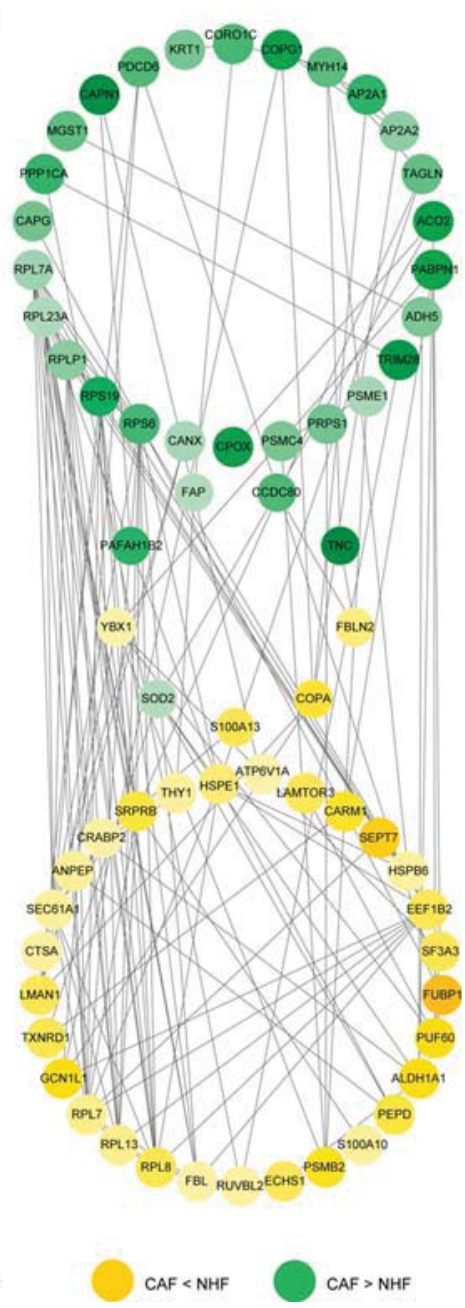

Figure 5. Comparison of proteomes of CAF and NHF gown in 3D. (A) Protein abundance plot comparing 3D-CAF and -NHF proteomes by a SILAC approach $(n=2)$. Significantly altered proteins were determined by performing outlier tests using Significance B values generated by MaxQuant $^{22}$ and Perseus. ${ }^{24}$ Regulated proteins are highlighted in blue $(p \leq 0.05)$ and in red $(p \leq 0.01)$, respectively. (B) STRING network analysis of significantly regulated proteins of CAF and NHF in 3D culture $(p<0.05)$. (C) GO-term enrichment $(p \leq 0.03$, colored circles) of significantly regulated proteins in $3 \mathrm{D}$ culture in $\mathrm{CAF}$ in comparison to NHF.

$\left.=4.8 \times 10^{-11}\right)$ and collagen fibril organization $(\mathrm{FDR}=8.62 \times$ $10^{-5}$ ) irrespectively of the used cell type (Figure $4 \mathrm{C}$ ). Among these ECM proteins, we identified several proteins that were previously shown to be involved in tumor progression. The proteoglycan biglycan (BGN) was found upregulated in CAF by increased TGF- $\beta 1$ and bFGF signaling ${ }^{38}$ and was shown to promote melanoma invasion. ${ }^{39}$ Also, the heparin-binding protein periostin (POSTN) was shown to be induced by bFGF signaling and to promote cancer cell survival. ${ }^{40}$

Taken together, 3D culture conditions significantly altered the protein compositions of cells and led to an upregulation of cellular proteins critical for the establishment and maintenance of ECM-cell contacts. Critically, 3D culture conditions might reveal differentially regulated proteins important in disease, which are not as such detectable or regulated under $2 \mathrm{D}$ culture conditions. To characterize potential CAF-specific mechanisms critical for ECM contraction, we performed a differential analysis comparing NHF and CAF proteomes of cells grown in 3D.
3D Culture Conditions Induce CAF-Specific Changes That Are Not Observable in 2D

Using our 3D-SILAC approach, we directly compared NHF and CAF grown in 3D by normalizing them to a common super-SILAC mix as described in Figure 1A. We identified $2^{\prime} 238$ proteins of which we could quantify 979 in two replicates each (Figure 5A, Supplementary Figure S5, Supplementary Table S4). In total, 94 proteins were significantly altered between NHF and CAF $(p \leq 0.05)$, of which 64 potentially interact according to STRING DB. Importantly, the network contained a cluster of differentially regulated ECM proteins, proteins associated with extracellular space and regulation of extracellular stimuli (Figure 5B, center circle). GO analysis of significantly regulated proteins showed an enrichment for proteins involved in vacuolar and lysosomal transport $(p=0.008)$, regulation of response to stress $(p=$ $0.009)$, multicellular organismal process $(p=0.0047)$, and cellular development and differentiation $(p=0.02$; Figure 5C, Supplementary Figure S6). Especially the latter two terms nicely reflect the influence of 3D culture conditions. By comparing our data sets with a published ex vivo analysis of breast cancer biopsies, it became evident that the $3 \mathrm{D}$ data set 


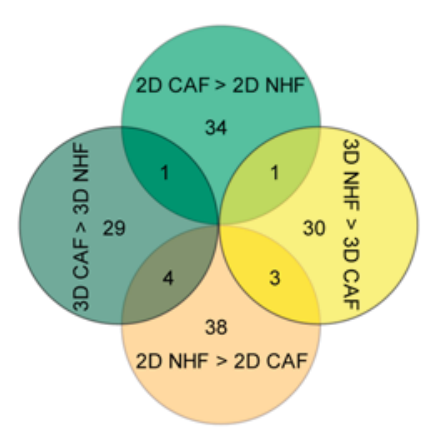

D

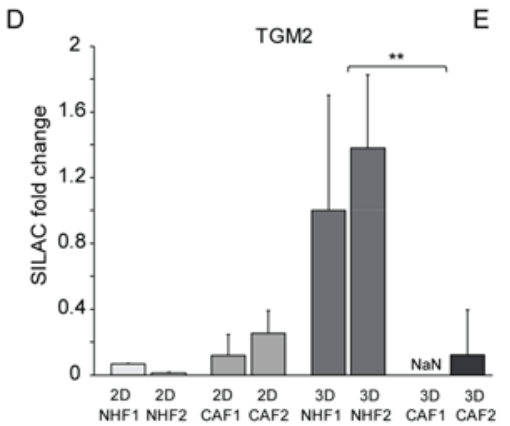

B

$E$
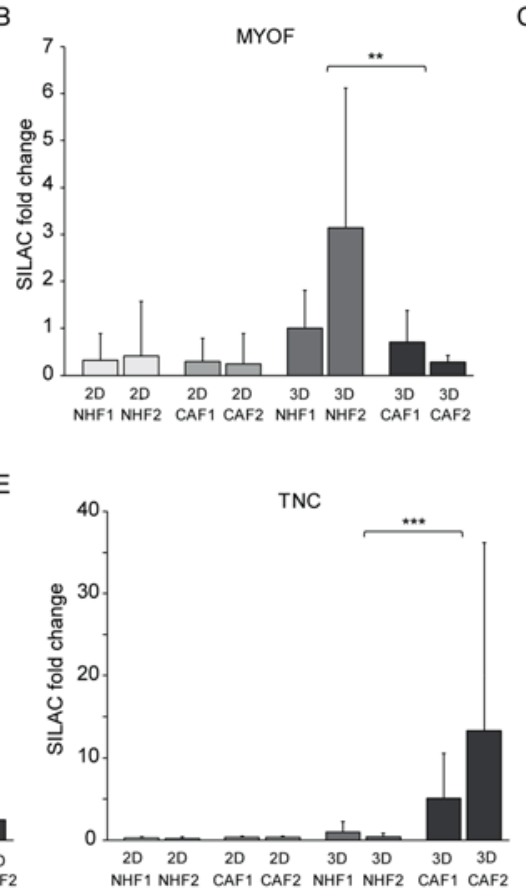
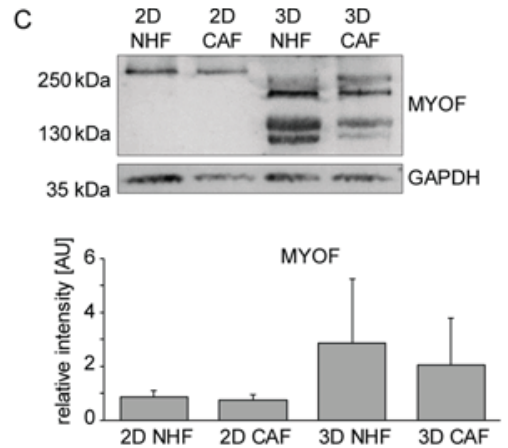

$\mathrm{F}$

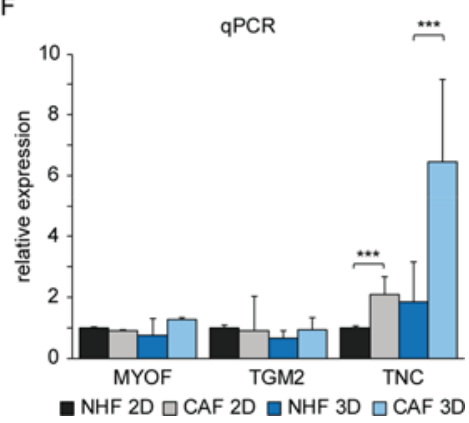

Figure 6. Differential abundances of proteins in $2 \mathrm{D}$ and $3 \mathrm{D}$ cultures. (A) Venn diagram of proteins that were identified as significantly regulated comparing proteomes of CAF and NHF in 2D or 3D (Significance B, $p<0.05$ ). (B) Normalized fold change of SILAC ratios of MYOF of cells grown in $2 \mathrm{D}$ and $3 \mathrm{D}$, normalized to $3 \mathrm{D}$ NHF1. Error bars indicate variability of respective peptide quantifications. Significance B was calculated with $p<0.05$ for the $\log _{2}$ ratios $2 \mathrm{D} \mathrm{CAF} / 2 \mathrm{D}$ NHF and the sum of all intensities in 2D, and with the $\log _{2}$ ratios of 3D CAF/3D NHF and the sum of all intensities in 3D. (C) Western blot of MYOF in 2D and 3D NHF and CAF. Quantification of the Western blot is shown in the lower panel ( $n$ =2). Normalized fold change of SILAC ratios of (D) TGM2 and (E) TNC of cells grown in 2D and 3D, normalized to 3D NHF1. Error bars indicate variability of respective peptide quantifications. Statistical analyses were performed as in panel (B). (F) Relative gene expression of MYOF, TGM2, and TNC under 2D and 3D culture conditions normalized to HPRT. Shown are ratios of biological replicates $(n=2$ with three technical replicates each). $* * * p \leq 0.001$.

correlated slightly better compared to the $2 \mathrm{D}$ data set (Pearson coefficient of 0.38 versus 0.32 (Supplementary Figure S7). ${ }^{41}$ Also, in 3D cultures, published CAF biomarkers better resembled in vivo observations. ${ }^{42}$ Out of $113 \mathrm{CAF}$ marker proteins, we identified 16 in $3 \mathrm{D}$ and 17 in $2 \mathrm{D}$, respectively. Critically, in $3 \mathrm{D}, 15$ out of 16 proteins were regulated as published, whereas in $2 \mathrm{D}$, only 7 out of 17 were regulated consistently (Supplementary Table S5). Thus, compared to 2D cultures, 3D culture systems appear to mimic closer observations made in vivo.

Interestingly, by comparing single proteins that were identified as significantly regulated in $3 \mathrm{D}$ or in $2 \mathrm{D}$, it became evident that only few proteins overlapped (Figure 6A, Supplementary Table S6). More importantly, some proteins were opposingly regulated: four proteins were significantly upregulated in CAF in $3 \mathrm{D}$, but in $2 \mathrm{D}$ they were upregulated in NHF. Among these proteins was TRIM28, a transcription factor known to be higher expressed in cancerous tissues ${ }^{43}$ and transgelin (TAGLN), an actin cross-linking protein, which mediates cell contractility and promotes migration and invasion of cancer stem cells. ${ }^{44}$ In $3 \mathrm{D}$, caveolin-1 (CAV-1) was decreased in CAF in comparison to NHF, whereas in $2 \mathrm{D}$, CAV-1 was higher expressed in CAF than in NHF. Decreased CAV-1 expression in CAF is known to promote cancer cell invasion and staining of breast cancer sections showed a clear decrease of CAV- 1 in $\alpha$-SMA positive cells. ${ }^{45}$

Myoferlin (MYOF) is a protein that we found significantly upregulated in 3D NHF in comparison to CAF by SILACbased MS $(p=0.004)$ as well as by Western blot, with no significant regulation in $2 \mathrm{D}$ (Figure 6B,C). MYOF is a $\mathrm{Ca}^{2+}$ regulated vesicular protein known to regulate intracellular trafficking of growth factor receptors. ${ }^{46,47}$ It was shown to be overexpressed in human breast cancers regulating growth factor receptor activation. Our results indicate that cancer cells themselves and not the tumor stroma overexpress MYOF implicating that MYOF plays distinct roles in cancer and stromal cells. Interestingly, MYOF was shown to be proteolytically cleaved in breast cancer and cell lines leading to increased ERK1/2 phosphorylation. ${ }^{48}$ We also observed different MYOF isoforms under $3 \mathrm{D}$ conditions by Western blot (Figure 6C). Their roles in fibroblasts and tumor stroma will have to be addressed in future studies.

Two proteins that are known to be important for CAF biology are tenascin (TNC) and transglutaminase 2 (TGM2) (Figure 6D,E). TNC, an ECM glycoprotein upregulated in wound healing and cancer which also influences gene expression, ${ }^{49}$ was highly upregulated under $3 \mathrm{D}$ culture conditions in $\operatorname{CAF}\left(p=1.8 \times 10^{-8}\right) .^{50,51}$ In $2 \mathrm{D}$ conditions, this difference was not observed. Interestingly, upregulation of TNC appeared to be regulated on gene expression level as indicated by increased mRNA levels (Figure 6F). In contrast, abundance of transglutaminase 2 (TGM2) was significantly elevated in NHF in comparison to CAF in $3 \mathrm{D}(p=0.01)$. In $2 \mathrm{D}$, no significant differences in TGM2 abundance could be observed, but CAF showed tendencies of higher TGM2 levels than NHF. TGM2 is among others critical for cross-linking of ECM proteins and ECM stability. ${ }^{52}$ Its roles in cancer are controversially discussed as it might have pro- and 
antiapoptotic properties. ${ }^{53}$ TGM2 absence in the stroma is thought to be a favoring condition for the formation and development of metastases, while TGM2 in tumor cells might be required for the development of metastases. ${ }^{54,55}$ In contrast to TNC, TGM2, and MYOF gene expression analyses could not be used to predict protein abundance (Figure 6F). mRNA and protein abundance anticorrelated indicating extensive posttranscriptional/post-translational regulatory mechanism.

\section{CONCLUSION}

In the current publication, we introduce a $3 \mathrm{D}$ cell culture method based on a mixture of collagen I and matrigel that is amenable to SILAC labeling and that allows an accurate quantitative analysis of intracellular proteins and the cell microenvironment. In addition, SILAC labeling supports the discrimination of cell-intrinsic from exogenous matrix proteins. Because of increased sample complexity, however, SILAC labeling might negatively influence identification rates. To increase identification rates, data-dependent as well as dataindependent label-free quantification approaches can be used in the future.

We highlight proteomic changes of cells, which are transferred from $2 \mathrm{D}$ to $3 \mathrm{D}$ and specifically address the response of CAF in 3D. Critically, 3D cell cultures highlight cell type-specific differences that are not observable in $2 \mathrm{D}$ and that closely mimic in vivo observations. Thus, our approach should alleviate the in vitro identification and characterization of in vivo-relevant biomarkers and new disease targets. Together with organoid cultures, (organotypic) 3D cell culture systems hold great promise for improved drug development, reducing the need for animal experiments and avoiding nonhuman species-specific effects.

\section{ASSOCIATED CONTENT}

\section{Supporting Information}

The Supporting Information is available free of charge on the ACS Publications website at DOI: 10.1021/acs.jproteome.8b00237.

Supplementary figures (PDF)

SILAC ratios of NHF and CAF from 2D culture (XLSX) Collagen I (Corning) and Matrigel (growth factor reduced, Corning) (XLSX)

SILAC ratios from $2 \mathrm{D}$ versus $3 \mathrm{D}$ culture conditions (XLSX)

SILAC ratios of NHF and CAF from 3D culture (XLSX)

Comparison of $\mathrm{CAF}$ consensus proteome to $2 \mathrm{D}$ versus 3D data (XLSX)

Significantly regulated proteins in $2 \mathrm{D}$ and $3 \mathrm{D}$ normalized to the super-SILAC mix (XLSX)

\section{AUTHOR INFORMATION}

\section{Corresponding Author}

*E-mail: joern.dengjel@unifr.ch. Phone: +41 263008631. ORCID $\odot$

Jörn Dengjel: 0000-0002-9453-4614

Notes

The authors declare no competing financial interest.

MS proteomics data were deposited to the ProteomeXchange Consortium via the $\mathrm{PRIDE}^{23}$ partner repository with the data set identifier PXD009424.

\section{ACKNOWLEDGMENTS}

The research leading to these results has received funding from the German Research Foundation (DFG) through CRC 850 project B8 and DE $1757 / 3-2$, and from the Swiss National Science Foundation, Grant No. 31003A-166482/1.

\section{REFERENCES}

(1) Langley, G. R.; Adcock, I. M.; Busquet, F.; Crofton, K. M.; Csernok, E.; Giese, C.; Heinonen, T.; Herrmann, K.; HofmannApitius, M.; Landesmann, B.; Marshall, L. J.; McIvor, E.; Muotri, A. R.; Noor, F.; Schutte, K.; Seidle, T.; van de Stolpe, A.; Van Esch, H.; Willett, C.; Woszczek, G. Towards a 21st-century roadmap for biomedical research and drug discovery: consensus report and recommendations. Drug Discovery Today 2017, 22 (2), 327-39.

(2) Eke, I.; Hehlgans, S.; Zong, Y.; Cordes, N. Comprehensive analysis of signal transduction in three-dimensional ECM-based tumor cell cultures. J. Biol. Methods 2015, 2 (4), 31.

(3) Herr, R.; Kohler, M.; Andrlova, H.; Weinberg, F.; Moeller, Y.; Halbach, S.; Lutz, L.; Mastroianni, J.; Klose, M.; Bittermann, N.; Kowar, S.; Zeiser, R.; Olayioye, M. A.; Lassmann, S.; Busch, H.; Boerries, M.; Brummer, T. B-Raf inhibitors induce epithelial differentiation in BRAF-mutant colorectal cancer cells. Cancer Res. 2015, 75 (1), 216-29.

(4) Martin, K. J.; Patrick, D. R.; Bissell, M. J.; Fournier, M. V. Prognostic breast cancer signature identified from 3D culture model accurately predicts clinical outcome across independent datasets. PLoS One 2008, 3 (8), e2994.

(5) Augsten, M. Cancer-associated fibroblasts as another polarized cell type of the tumor microenvironment. Front. Oncol. 2014, 4, 62.

(6) Goetz, J. G.; Minguet, S.; Navarro-Lerida, I.; Lazcano, J. J.; Samaniego, R.; Calvo, E.; Tello, M.; Osteso-Ibanez, T.; Pellinen, T.; Echarri, A.; Cerezo, A.; Klein-Szanto, A. J.; Garcia, R.; Keely, P. J.; Sanchez-Mateos, P.; Cukierman, E.; Del Pozo, M. A. Biomechanical remodeling of the microenvironment by stromal caveolin-1 favors tumor invasion and metastasis. Cell 2011, 146 (1), 148-63.

(7) Gaggioli, C.; Hooper, S.; Hidalgo-Carcedo, C.; Grosse, R.; Marshall, J. F.; Harrington, K.; Sahai, E. Fibroblast-led collective invasion of carcinoma cells with differing roles for RhoGTPases in leading and following cells. Nat. Cell Biol. 2007, 9 (12), 1392-400.

(8) Cirri, P.; Chiarugi, P. Cancer associated fibroblasts: the dark side of the coin. Am. J. Cancer Res. 2011, 1 (4), 482-97.

(9) Chang, H. Y.; Sneddon, J. B.; Alizadeh, A. A.; Sood, R.; West, R. B.; Montgomery, K.; Chi, J. T.; van de Rijn, M.; Botstein, D.; Brown, P. O. Gene expression signature of fibroblast serum response predicts human cancer progression: similarities between tumors and wounds. PLoS Biol. 2004, 2 (2), E7.

(10) Matsuo, Y.; Ochi, N.; Sawai, H.; Yasuda, A.; Takahashi, H.; Funahashi, H.; Takeyama, H.; Tong, Z.; Guha, S. CXCL8/IL-8 and CXCL12/SDF-1alpha co-operatively promote invasiveness and angiogenesis in pancreatic cancer. Int. J. Cancer 2009, 124 (4), 853-61.

(11) Yue, X.; Lukowski, J. K.; Weaver, E. M.; Skube, S. B.; Hummon, A. B. Quantitative Proteomic and Phosphoproteomic Comparison of 2D and 3D Colon Cancer Cell Culture Models. J. Proteome Res. 2016, 15 (12), 4265-76.

(12) He, W.; Kuang, Y.; Xing, X.; Simpson, R. J.; Huang, H.; Yang, T.; Chen, J.; Yang, L.; Liu, E.; He, W.; Gu, J. Proteomic comparison of $3 \mathrm{D}$ and $2 \mathrm{D}$ glioma models reveals increased HLA-E expression in 3D models is associated with resistance to NK cell-mediated cytotoxicity. J. Proteome Res. 2014, 13 (5), 2272-81.

(13) Chan, Y. H.; Huang, T. W.; Young, T. H.; Lou, P. J. Human salivary gland acinar cells spontaneously form three-dimensional structures and change the protein expression patterns. J. Cell. Physiol. 2011, 226 (11), 3076-85.

(14) Rigbolt, K. T.; Blagoev, B. Proteome-wide quantitation by SILAC. Methods Mol. Biol. 2010, 658, 187-204.

(15) Zanivan, S.; Maione, F.; Hein, M. Y.; Hernandez-Fernaud, J. R.; Ostasiewicz, P.; Giraudo, E.; Mann, M. SILAC-based proteomics of 
human primary endothelial cell morphogenesis unveils tumor angiogenic markers. Mol. Cell. Proteomics 2013, 12 (12), 3599-611.

(16) Hughes, C.; Radan, L.; Chang, W. Y.; Stanford, W. L.; Betts, D. H.; Postovit, L. M.; Lajoie, G. A. Mass spectrometry-based proteomic analysis of the matrix microenvironment in pluripotent stem cell culture. Mol. Cell. Proteomics 2012, 11 (12), 1924-36.

(17) Gonneaud, A.; Jones, C.; Turgeon, N.; Levesque, D.; Asselin, C.; Boudreau, F.; Boisvert, F. M. A SILAC-Based Method for Quantitative Proteomic Analysis of Intestinal Organoids. Sci. Rep. 2016, 6, 38195.

(18) Mittapalli, V. R.; Madl, J.; Loffek, S.; Kiritsi, D.; Kern, J. S.; Romer, W.; Nystrom, A.; Bruckner-Tuderman, L. Injury-Driven Stiffening of the Dermis Expedites Skin Carcinoma Progression. Cancer Res. 2016, 76 (4), 940-51.

(19) Sprenger, A.; Weber, S.; Zarai, M.; Engelke, R.; Nascimento, J. M.; Gretzmeier, C.; Hilpert, M.; Boerries, M.; Has, C.; Busch, H.; Bruckner-Tuderman, L.; Dengjel, J. Consistency of the proteome in primary human keratinocytes with respect to gender, age, and skin localization. Mol. Cell. Proteomics 2013, 12 (9), 2509-21.

(20) Camfield, C. S.; Camfield, P. R.; Finley, J. Screening of subsequent siblings of children who die of sudden infant death syndrome. Am. J. Dis Child 1987, 141 (7), 807-10.

(21) Wozniak, M. A.; Keely, P. J. Use of three-dimensional collagen gels to study mechanotransduction in T47D breast epithelial cells. Biol. Proced. Online 2005, 7, 144-61.

(22) Cox, J.; Mann, M. MaxQuant enables high peptide identification rates, individualized p.p.b.-range mass accuracies and proteome-wide protein quantification. Nat. Biotechnol. 2008, 26 (12), $1367-72$.

(23) Vizcaino, J. A.; Csordas, A.; del-Toro, N.; Dianes, J. A.; Griss, J.; Lavidas, I.; Mayer, G.; Perez-Riverol, Y.; Reisinger, F.; Ternent, T.; $\mathrm{Xu}$, Q. W.; Wang, R.; Hermjakob, H. 2016 update of the PRIDE database and its related tools. Nucleic Acids Res. 2016, 44 (D1), D447-56.

(24) Tyanova, S.; Temu, T.; Sinitcyn, P.; Carlson, A.; Hein, M. Y.; Geiger, T.; Mann, M.; Cox, J. The Perseus computational platform for comprehensive analysis of (prote)omics data. Nat. Methods 2016, 13 (9), $731-40$.

(25) Jensen, L. J.; Kuhn, M.; Stark, M.; Chaffron, S.; Creevey, C.; Muller, J.; Doerks, T.; Julien, P.; Roth, A.; Simonovic, M.; Bork, P.; von Mering, C. STRING 8-a global view on proteins and their functional interactions in 630 organisms. Nucleic Acids Res. 2009, 37 (Database), D412-6.

(26) Shannon, P.; Markiel, A.; Ozier, O.; Baliga, N. S.; Wang, J. T.; Ramage, D.; Amin, N.; Schwikowski, B.; Ideker, T. Cytoscape: a software environment for integrated models of biomolecular interaction networks. Genome Res. 2003, 13 (11), 2498-504.

(27) Maere, S.; Heymans, K.; Kuiper, M. BiNGO: a Cytoscape plugin to assess overrepresentation of gene ontology categories in biological networks. Bioinformatics 2005, 21 (16), 3448-9.

(28) Albrengues, J.; Bourget, I.; Pons, C.; Butet, V.; Hofman, P.; Tartare-Deckert, S.; Feral, C. C.; Meneguzzi, G.; Gaggioli, C. LIF mediates proinvasive activation of stromal fibroblasts in cancer. Cell Rep. 2014, 7 (5), 1664-78.

(29) Geiger, T.; Cox, J.; Ostasiewicz, P.; Wisniewski, J. R.; Mann, M. Super-SILAC mix for quantitative proteomics of human tumor tissue. Nat. Methods 2010, 7 (5), 383-5.

(30) Iyer, V. R.; Eisen, M. B.; Ross, D. T.; Schuler, G.; Moore, T.; Lee, J. C.; Trent, J. M.; Staudt, L. M.; Hudson, J., Jr.; Boguski, M. S.; Lashkari, D.; Shalon, D.; Botstein, D.; Brown, P. O. The transcriptional program in the response of human fibroblasts to serum. Science 1999, 283 (5398), 83-7.

(31) Szklarczyk, D.; Morris, J. H.; Cook, H.; Kuhn, M.; Wyder, S.; Simonovic, M.; Santos, A.; Doncheva, N. T.; Roth, A.; Bork, P.; Jensen, L. J.; von Mering, C. The STRING database in 2017: qualitycontrolled protein-protein association networks, made broadly accessible. Nucleic Acids Res. 2017, 45 (D1), D362-D8.

(32) Labernadie, A.; Kato, T.; Brugues, A.; Serra-Picamal, X.; Derzsi, S.; Arwert, E.; Weston, A.; Gonzalez-Tarrago, V.; Elosegui-Artola, A.;
Albertazzi, L.; Alcaraz, J.; Roca-Cusachs, P.; Sahai, E.; Trepat, X. A mechanically active heterotypic E-cadherin/N-cadherin adhesion enables fibroblasts to drive cancer cell invasion. Nat. Cell Biol. 2017, 19 (3), 224-37.

(33) Kramer, N.; Schmollerl, J.; Unger, C.; Nivarthi, H.; Rudisch, A.; Unterleuthner, D.; Scherzer, M.; Riedl, A.; Artaker, M.; Crncec, I.; Lenhardt, D.; Schwarz, T.; Prieler, B.; Han, X.; Hengstschlager, M.; Schuler, J.; Eferl, R.; Moriggl, R.; Sommergruber, W.; Dolznig, H. Autocrine WNT2 signaling in fibroblasts promotes colorectal cancer progression. Oncogene 2017, 36 (39), 5460-72.

(34) Tomasek, J. J.; Haaksma, C. J.; Eddy, R. J.; Vaughan, M. B. Fibroblast contraction occurs on release of tension in attached collagen lattices: dependency on an organized actin cytoskeleton and serum. Anat. Rec. 1992, 232 (3), 359-68.

(35) Bonan, S.; Albrengues, J.; Grasset, E.; Kuzet, S. E.; Nottet, N.; Bourget, I.; Bertero, T.; Mari, B.; Meneguzzi, G.; Gaggioli, C. Membrane-bound ICAM-1 contributes to the onset of proinvasive tumor stroma by controlling acto-myosin contractility in carcinomaassociated fibroblasts. Oncotarget 2017, 8 (1), 1304-20.

(36) Hooper, S.; Gaggioli, C.; Sahai, E. A chemical biology screen reveals a role for Rab21-mediated control of actomyosin contractility in fibroblast-driven cancer invasion. Br. J. Cancer 2010, 102 (2), 392402.

(37) Sprenger, A.; Kuttner, V.; Bruckner-Tuderman, L.; Dengjel, J. Global proteome analyses of SILAC-labeled skin cells. Methods Mol. Biol. 2013, 961, 179-91.

(38) Van Bockstal, M.; Lambein, K.; Van Gele, M.; De Vlieghere, E.; Limame, R.; Braems, G.; Van den Broecke, R.; Cocquyt, V.; Denys, H.; Bracke, M.; Libbrecht, L.; De Wever, O. Differential regulation of extracellular matrix protein expression in carcinoma-associated fibroblasts by TGF-beta1 regulates cancer cell spreading but not adhesion. Oncoscience 2014, 1 (10), 634-48.

(39) Andrlova, H.; Mastroianni, J.; Madl, J.; Kern, J. S.; Melchinger, W.; Dierbach, H.; Wernet, F.; Follo, M.; Technau-Hafsi, K.; Has, C.; Rao Mittapalli, V.; Idzko, M.; Herr, R.; Brummer, T.; Ungefroren, H.; Busch, H.; Boerries, M.; Narr, A.; Ihorst, G.; Vennin, C.; SchmittGraeff, A.; Minguet, S.; Timpson, P.; Duyster, J.; Meiss, F.; Romer, W.; Zeiser, R. Biglycan expression in the melanoma microenvironment promotes invasiveness via increased tissue stiffness inducing integrin-beta1 expression. Oncotarget 2017, 8 (26), 42901-16.

(40) Ouyang, G.; Liu, M.; Ruan, K.; Song, G.; Mao, Y.; Bao, S. Upregulated expression of periostin by hypoxia in non-small-cell lung cancer cells promotes cell survival via the Akt/PKB pathway. Cancer Lett. 2009, 281 (2), 213-9.

(41) Groessl, M.; Slany, A.; Bileck, A.; Gloessmann, K.; Kreutz, D.; Jaeger, W.; Pfeiler, G.; Gerner, C. Proteome profiling of breast cancer biopsies reveals a wound healing signature of cancer-associated fibroblasts. J. Proteome Res. 2014, 13 (11), 4773-82.

(42) Santi, A.; Kugeratski, F. G.; Zanivan, S. Cancer Associated Fibroblasts: The Architects of Stroma Remodeling. Proteomics 2018, 18 (5-6), e1700167.

(43) Liu, L.; Zhao, E.; Li, C.; Huang, L.; Xiao, L.; Cheng, L.; Huang, $\mathrm{X}$.; Song, Y.; Xu, D. TRIM28, a new molecular marker predicting metastasis and survival in early-stage non-small cell lung cancer. Cancer Epidemiol. 2013, 37 (1), 71-8.

(44) Lee, E. K.; Han, G. Y.; Park, H. W.; Song, Y. J.; Kim, C. W. Transgelin promotes migration and invasion of cancer stem cells. J. Proteome Res. 2010, 9 (10), 5108-17.

(45) Simpkins, S. A.; Hanby, A. M.; Holliday, D. L.; Speirs, V. Clinical and functional significance of loss of caveolin-1 expression in breast cancer-associated fibroblasts. J. Pathol 2012, 227 (4), 490-8.

(46) Bernatchez, P. N.; Acevedo, L.; Fernandez-Hernando, C.; Murata, T.; Chalouni, C.; Kim, J.; Erdjument-Bromage, H.; Shah, V.; Gratton, J. P.; McNally, E. M.; Tempst, P.; Sessa, W. C. Myoferlin regulates vascular endothelial growth factor receptor-2 stability and function. J. Biol. Chem. 2007, 282 (42), 30745-53.

(47) Turtoi, A.; Blomme, A.; Bellahcene, A.; Gilles, C.; Hennequiere, V.; Peixoto, P.; Bianchi, E.; Noel, A.; De Pauw, E.; Lifrange, E.; 
Delvenne, P.; Castronovo, V. Myoferlin is a key regulator of EGFR activity in breast cancer. Cancer Res. 2013, 73 (17), 5438-48.

(48) Piper, A. K.; Ross, S. E.; Redpath, G. M.; Lemckert, F. A.; Woolger, N.; Bournazos, A.; Greer, P. A.; Sutton, R. B.; Cooper, S. T. Enzymatic cleavage of myoferlin releases a dual C2-domain module linked to ERK signalling. Cell. Signalling 2017, 33, 30-40.

(49) Midwood, K. S.; Chiquet, M.; Tucker, R. P.; Orend, G. Tenascin-C at a glance. J. Cell Sci. 2016, 129 (23), 4321-7.

(50) Ishii, K.; Mizokami, A.; Tsunoda, T.; Iguchi, K.; Kato, M.; Hori, Y.; Arima, K.; Namiki, M.; Sugimura, Y. Heterogenous induction of carcinoma-associated fibroblast-like differentiation in normal human prostatic fibroblasts by co-culturing with prostate cancer cells. J. Cell. Biochem. 2011, 112 (12), 3604-11.

(51) Naba, A.; Clauser, K. R.; Lamar, J. M.; Carr, S. A.; Hynes, R. O. Extracellular matrix signatures of human mammary carcinoma identify novel metastasis promoters. eLife 2014, 3, e01308.

(52) Kuttner, V.; Mack, C.; Gretzmeier, C.; Bruckner-Tuderman, L.; Dengjel, J. Loss of collagen VII is associated with reduced transglutaminase 2 abundance and activity. J. Invest. Dermatol. 2014, 134 (9), 2381-9.

(53) Tatsukawa, H.; Furutani, Y.; Hitomi, K.; Kojima, S. Transglutaminase 2 has opposing roles in the regulation of cellular functions as well as cell growth and death. Cell Death Dis. 2016, 7 (6), e2244.

(54) Di Giacomo, G.; Lentini, A.; Beninati, S.; Piacentini, M.; Rodolfo, C. In vivo evaluation of type 2 transglutaminase contribution to the metastasis formation in melanoma. Amino Acids 2009, 36 (4), $717-24$.

(55) Jones, R. A.; Kotsakis, P.; Johnson, T. S.; Chau, D. Y.; Ali, S.; Melino, G.; Griffin, M. Matrix changes induced by transglutaminase 2 lead to inhibition of angiogenesis and tumor growth. Cell Death Differ. 2006, 13 (9), 1442-53. 